\title{
Uterine Leiomyosarcoma: A Case Report
}

\author{
Forough Forghani ${ }^{1}$, Abolfazl Mehdizadeh Kashi $^{2}$, Kambiz Sadegi $^{3,4}$, Mania Kaveh $^{2,{ }^{*} \mathbb{C}}$, \\ Mehrangiz Ghafari ${ }^{5}$
}

\begin{abstract}
Objectives: Uterine leiomyosarcoma (LMS) is a rare cancer originated from smooth muscle lining the walls of the uterus. LMS is known as an aggressive tumor with high mortality and morbidity rates compared to other uterine cancers, despite the disease stage at the time of diagnosis. In most cases, LMS has been misdiagnosed as benign uterine leiomyoma following hysterectomy or myomectomy.

Case Presentation: We present a 53-year-old G7 L7 woman who was referred to our clinic for abnormal uterine bleeding (AUB) for 6 months. On physical examination, we found an abdominal mass that had grown rapidly in the last 4 months. The computed tomography (CT) scan results showed a heterogeneous mass extending from the epigastric region to the pelvic area. Following an exploratory laparotomy, histopathology report confirmed the diagnosis of LMS. Her uterus, Fallopian tubes and ovaries were removed during a surgery, and she was referred to a gynecologic oncologist for possible chemotherapy.

Conclusions: We found that the surgery was the only treatment for LMS. Although there is a faint possibility to diagnose LMS before surgery, in the patient with uncertain diagnosis and suspicious of LMS, analysis of LDH and LDH3 levels along with dynamic gadolinium- diethylenetriamine penta-acetic acid (Gd-DTPA) enhanced magnetic resonance imaging (MRI) is recommended.

Keywords: Uterine leiomyosarcoma, Gynecologic oncologist
\end{abstract}

\section{Introduction}

Uterine leiomyosarcoma (LMS) is a rare cancer originated from smooth muscle lining the walls of the uterus with a high risk of development and recurrence. LMS is responsible for $8 \%$ of uterine malignancies (1). About $42 \%-60 \%$ of uterine sarcoma cases are related to various subtypes of LMS. Abnormal uterine bleeding (AUB) as well as pelvic pressure, pain and mass are considered as the first findings and symptoms of leiomyosarcomas, which make it difficult to distinguish between them (2-4). LMS is usually diagnosed following a surgery, although in a rare condition, frozen section analysis is used during a surgery. Grade of the tumor and histopathological findings help physicians to choose the right clinical approach and treatment modality. There is no single reliable test to apply before the surgery to distinguish between benign or malignant uterine disease. We used computed tomography (CT) scan results and histopathology report following an exploratory laparotomy to diagnose LMS in a 53-year-old G7 L7 woman who complained of AUB and rapidly growing abdominal mass. This review is a case report discussing uterine leiomyosarcoma, the clinical characteristics, diagnosis, outcome and recent advances.

\section{Case Report}

A 53-year-old G7 L7 woman living in Zabol, Sistan and
Baluchestan province, Iran, was suffering from AUB for 6 months. She was admitted to the emergency room of Zabol hospital, Zabol, Iran, with complaints of heavy vaginal bleeding and passing blood clots in January 2016. She had mild to moderate pain in the lower abdomen toward the epigastric area. The patient noticed a rapidly growing mass in the lower abdomen from 4 months ago. Patient also complained of abdominal bloating and loss of appetite. There was no symptom of painful bladder or bowl syndromes. Furthermore, there was no history of chronic or long-term diseases.

\begin{abstract}
Physical Examination
On examination, patient looked pale and had stable vital signs with blood pressure (BP) of $120 / 79 \mathrm{~mm} \mathrm{Hg}$, pulse rate (PR) of 96 pulses/second and respiratory rate (RR) of 18 breaths/minute. On the abdominal examination, a soft to firm, non-tender mass was palpated in midline abdominal area, spreading from lower abdomen to epigastric area. On vaginal examination, there was an enlarged anteverted uterus, like the size of 20 week pregnancy. There was no cervical motion tenderness (CMT) and no bilateral tenderness on fornix palpation.
\end{abstract}

\section{Investigation}

The CT scan results showed a heterogeneous mass with

Received 2 April 2017, Accepted 11 November 2017, Available online 26 November 2017

${ }^{1}$ Department of Obstetrics and Gynecology, Zabol University of Medical Sciences, Zabol, Iran. ${ }^{2}$ Endometriosis and Gynecological Disorder Research Center, Iran University of Medical Sciences, Tehran, Iran. ${ }^{3}$ Pain Research Center, Iran University of Medical Sciences, Tehran, Iran. ${ }^{4}$ Department of Anesthesiology, Zabol University of Medical Sciences, Zabol, Iran. ${ }^{5}$ Department of Pathology, Zabol University of Medical Sciences, Zabol, Iran.

*Corresponding Author: Mania Kaveh, Tel: xxx, Email: maniakaveh@gmail.com 
regions of multilocular and multiseptated mass and with approximate size of $93 \times 121 \mathrm{~cm}$, extending from pelvic area to epigastric area. The results also indicated that the mass possibly had spread to the uterine body and anterior wall of myometrium while connecting to the uterus.

Due to the pressure of the mass, the ovaries could not be checked. Free fluid was observed in the abdominal cavity. Muscle tissue and bone of hip region were normal and there was no sign of lymphadenopathy. Furthermore, the results of endometrial biopsy, performed on the patient in October 2015, indicated no presence of malignancy. At the same time, the report of Pap smear indicated chronic cervicitis, and in CBC diff, $\mathrm{Hb}$ was 11 . Therefore, based on these findings and vaginal bleeding from which the patient was suffering, a surgery was performed on the patient in January 2016. During surgery, an enlarged uterus, like the size of 20-week pregnancy, was observed, while there was a connection of dense adhesions of the omentum to mass and anterior abdominal wall. The mass arose from the uterine fundus originated from peritoneum and lobulated omentum (Figure 1). The samples from mass along with solid components, cystic and hemorrhage were then sent to the cytology laboratory of the hospital. Uterus, Fallopian tubes and ovaries were removed during the surgery, and the patient received 3 units of packed red blood cells (RBC).

\section{Macroscopic Features of Mass}

The dissection of enlarged uterus revealed an oval-shaped mass with the size of $27 \times 20 \times 15 \mathrm{~cm}$ and diameter of 11 $\mathrm{cm}$ inside the uterus cavity that had spread throughout the cervix. The cross-section of mass showed a gray-white, fleshy, and lobulated cut surface that contained foci of hemorrhage.

\section{Microscopic Features of Mass}

Evaluation of the issue sections showed spindle cells containing hyperchromatic nuclei and eosinophilic cytoplasm. The taken images indicated mitotic $(\mathrm{M})$ phase containing atypical mitosis (high-power fields [HPF] $>10 / 10$ ) and foci of necrosis. The total findings suggested LMS with cervix involvement. Peritoneal washings (PW) and cytologic analysis indicated no malignant cells.

There was no post-operative complication. The patient was discharged 7 days after the operation in satisfactory condition. After pathology report, the patient was referred to a gynecologic oncologist for possible chemotherapy (Figure $2 \mathrm{a}-\mathrm{e}$ ).

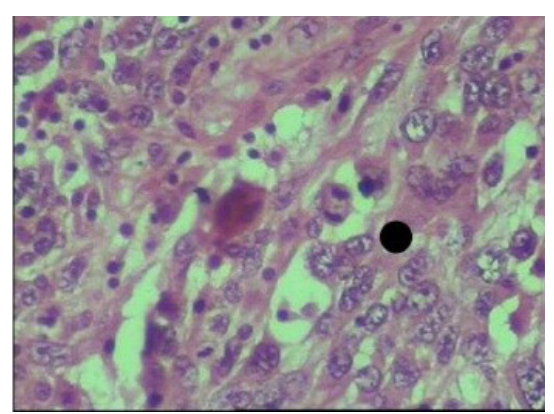

Figure 1. Atypical Mitotic Image.

\section{Discussion}

LMS is considered and is known as an aggressive tumor with high mortality and morbidity $(1,5)$. There is no reliable diagnostic method to distinguish between uterine LMS and benign uterine tumors before surgery. However, due to increased levels of total lactate dehydrogenase $(\mathrm{LDH})$ and $\mathrm{LDH}$ isoenzyme-3 in the patients with LMS, biochemical evaluations are also considered as a useful method. Among imaging studies, a high vascularity score in Doppler ultrasonography and high signal intensity in diffusion weighted imaging (DWI) also indicate malignant ovarian tumors (6). Diagnosis of LMS and determination of its stage are only based on histopathology report after surgery. Surgical staging of LMS includes hysterectomy, and bilateral salpingo-oophorectomy (BSO). The tumor larger than $5 \mathrm{~cm}$ and high mitotic index are important prognostic factors (7). Hematogenous spread is the most common form, and lymphatic spread is the rare one. Sarcoma is known as an aggressive tumor, meaning that there is still a high risk of local and distant recurrence after the tumor is removed(5). Stages I and II LMS, according to the International Federation of Gynecology and Obstetrics (FIGO) staging systems, have a very high risk of relapse, while its survival rate is also poor after relapse. One study indicated that the survival rates of LMS I and II are $50 \%$ and $25 \%$, respectively (8). Site of metastasis or recurrence is often around the liver and lungs, which is due to hematogenous spread (9). The patients with early stage LMS do not really undergo radiation therapy, and there is no overall survival benefit regarding radiation therapy. Some prospective studies have shown the effectiveness of chemotherapy in the treatment of uterine LMS(10). In our case due to heavy bleeding, we had no time for doing further diagnostic evaluation. Therefore we decided for surgery. Considering the macroscopic and microscopic features, leiomyosarcoma was diagnosed in
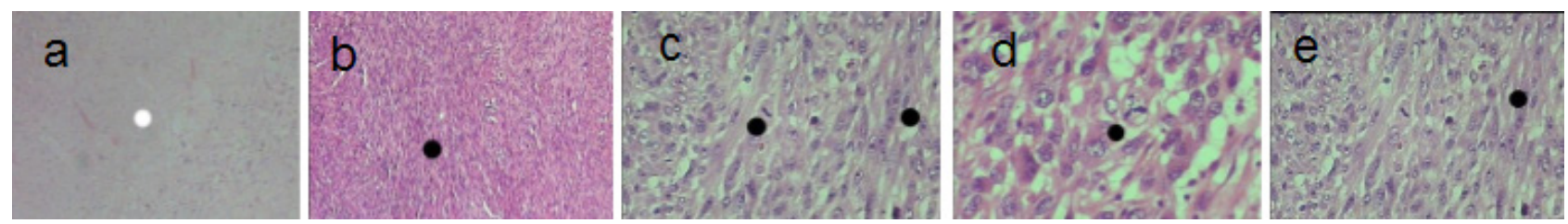

Figure 2. (a) foci of coagulative tumor cell necrosis. (b) Spindle cells arranged in intercalating bundle. (c) Pronounced nuclear pleomorphism. (d) pronounced nuclear pleomorphism. (e) nuclear pleomorphism and few mitotic images. 
the patient and after surgery, the patient was referred to a gynecologic oncologist.

\section{Conclusions}

Preoperative algorithm (low risk or high risk) should be aimed in order to avoid the occurrence of unexpected LMS diagnosed on the pathologic post-operative examination and selection of surgery. This should be done in the patients with history of myoma or those highly suspicious to LMS. Clinical and biochemical and ultrasonographic findings suspected of LMS and, only in highly suspected cases, a pelvic MRI could be initiated. And the surgery is the only treatment for leiomyosarcoma.

\section{Conflict of Interests}

Authors declare that they have no conflict of interests.

\section{Financial Support}

This study was financially supported by Iran University of Medical Sciences and Endometriosis and Gynecological Disorder Research Center.

\section{References}

1. Brooks SE, Zhan M, Cote T, Baquet CR. Surveillance, epidemiology, and end results analysis of 2677 cases of uterine sarcoma 1989-1999. Gynecol Oncol. 2004;93(1):204208. doi:10.1016/j.ygyno.2003.12.029

2. Baird DD, Dunson DB, Hill MC, Cousins D, Schectman JM. High cumulative incidence of uterine leiomyoma in black and white women: ultrasound evidence. Am J Obstet Gynecol. 2003;188(1):100-107.
3. Buttram VC Jr, Reiter RC. Uterine leiomyomata: etiology, symptomatology, and management. Fertil Steril. 1981;36(4):433-445.

4. Serden SP, Brooks PG. Treatment of abnormal uterine bleeding with the gynecologic resectoscope. J Reprod Med. 1991;36(10):697-699.

5. Kaur K, Kaur P, Kaur A, Singla A. Uterine leiomyosarcoma: A case report. J Midlife Health. 2014;5(4):202-204. doi:10.4103/0976-7800.145175

6. Goto A, Takeuchi S, Sugimura K, Maruo T. Usefulness of Gd-DTPA contrast-enhanced dynamic MRI and serum determination of $\mathrm{LDH}$ and its isozymes in the differential diagnosis of leiomyosarcoma from degenerated leiomyoma of the uterus. Int J Gynecol Cancer. 2002;12(4):354-361.

7. Sagae S, Yamashita K, Ishioka S, et al. Preoperative diagnosis and treatment results in 106 patients with uterine sarcoma in Hokkaido, Japan. Oncology. 2004;67(1):33-39. doi:10.1159/000080283

8. Abeler VM, Royne O, Thoresen S, Danielsen HE, Nesland JM, Kristensen GB. Uterine sarcomas in Norway. A histopathological and prognostic survey of a total population from 1970 to 2000 including 419 patients. Histopathology. 2009;54(3):355-364. doi:10.1111/j.13652559.2009.03231.x

9. Wright JD, Seshan VE, Shah M, et al. The role of radiation in improving survival for early-stage carcinosarcoma and leiomyosarcoma. Am J Obstet Gynecol. 2008;199(5):536. e531-538. doi:10.1016/j.ajog.2008.04.019

10. Hensley ML, Wathen JK, Maki RG, et al. Adjuvant therapy for high-grade, uterus-limited leiomyosarcoma: results of a phase 2 trial (SARC 005). Cancer. 2013;119(8):1555-1561. doi: $10.1002 /$ cncr. 27942

(C) 2018 The Author (s); This is an open-access article distributed under the terms of the Creative Commons Attribution License (http://creativecommons.org/licenses/by/4.0), which permits unrestricted use, distribution, and reproduction in any medium, provided the original work is properly cited. 